\title{
The Cost-effective Usefulness of Oral Iron Absorption Test- Prospective Evaluation in Premenopausal Women with Newly Diagnosed Iron Deficiency Anemia
}

\author{
Islam MS ${ }^{12 *}$, Dayley $\mathbf{D}^{\mathbf{1}}$ and Thanigaikumar $\mathbf{M}^{\mathbf{2}}$ \\ ${ }^{1}$ Department of Haematology, Broomfield Hospital, UK \\ ${ }^{2}$ Department of Haematology, Lewisham Hospital, UK \\ *Corresponding author: Islam MS, Department \\ of Haematology, Broomfield Hospital, CM1 7ET, \\ Chelmsford, UK
}

Received: April 02, 2018; Accepted: May 07, 2018; Published: May 21, 2018

\begin{abstract}
Background: We explored the cost-effective usefulness of oral iron absorption test (OIAT) to predict gastrointestinal lesions associated with iron malabsorption in premenopausal iron deficient women.

Materials and Methods: 238 premenopausal women referred to the haematology for evaluation of Iron deficiency anemia (IDA) were prospectively recruited by consecutive sampling. Patients with an identifiable cause of iron deficiency were not enrolled in the study. If any patient with abnormal OIAT was further investigated with IgA anti-TTG, IgG H pylori and IgG anti G-PCA antibody levels and 36 of those patients with abnormal OIAT also had gastrointestinal endoscopy and biopsy.
\end{abstract}

Results: Among the 238 study population 85 patients had an abnormal test. A high prevalence of celiac disease (7.5\%), $H$ pylori positivity $(62 \%)$ and autoimmune gastritis $(9.4 \%)$ was found among iron deficient women who had an abnormal OIAT. No significant difference was found in the occurrence of these gastrointestinal conditions with respect to menstrual flow. All patients who had a normal OIAT had a successful response to therapeutic iron supplementation. The OIAT based approach is 17 -fold more economical compared to blanket approach which include serological tests and endoscopy and biopsy in all patients.

Conclusion: OIAT is a useful simple inexpensive test which helps to target the appropriate patients for invasive and expensive tests for the evaluation of unexplained iron deficiency anemia.

Keywords: Iron deficiency anemia; Oral iron absorption test; Premenopausal; Celiac disease; $H$ pylori

\section{Abbreviations}

OIAT: Oral Iron Absorption Test; IDA: Iron Deficiency Anemia; G-PCA: Tissue Transglutaminase, Gastric Parietal Cell Antibody; DALYs: Disability-Adjusted Life-Years; GI: Gastrointestinal; $H$ pylori: Helicobacter Pylori; CRP: C-Reactive Protein; ESR: Erythrocyte Sedimentation Rate; MVC: Mean Corpuscular Volume; Hb: Hemoglobin; FBC: Full Blood Counts; EDTA: Ethylene Di-amine Tetra-acetic Acid; ELISA: Enzyme-Linked Immunosorbent Assay

\section{Introduction}

The prevalence of Iron deficiency anemia is very common as 30\%$50 \%$ of anemia is caused by iron deficiency [1]. Though the full global extent of iron deficiency is unknown, nearly two billion people suffer from anemia [2] roughly half of which is assumed to be attributable to a lack of adequate iron [3] the most common cause globally [4]. Iron deficiency is also implicated in nearly 200,000 deaths and 45 million disability-adjusted life-years (DALYs) lost annually (4.5\% of all risk-attributable DALYs) in the most recent 2013 Global Burden of Disease Study, predominantly due to iron deficiency anemia [5]. Furthermore, iron deficiency in the absence of anemia has been linked to many detrimental outcomes: lowered cognitive ability, reduced work capability, and greater rate of maternal and child mortality [6].

In the western world, IDA is prevalent in $5 \%$ of women aged $<50$ years [7]. Premenopausal women constitute a significant proportion of patients with unexplained IDA referred to hematologists. The evaluation of iron deficiency in the absence of evidence of excessive blood loss is often difficult. A popular clinical approach in such patients is to perform gastrointestinal (GI) endoscopy and at times more sophisticated tests like video capsule endoscopy, mesenteric angiography or isotope red cell scan, to identify occult GI hemorrhage.

The diagnostic pathway for young women affected by IDA is not clearly established. The British Society for Gastroenterology recommends gastroscopy for iron deficient women younger than 45 years only in the presence of GI symptoms [8]. However, symptoms are often mild and patients often refuse to undergo the invasive procedure. A large study which evaluated gastroscopy in patients with IDA concluded that it was unnecessary in about a third of premenopausal women [9].

Previous studies have shown that regardless of the menstrual flow, non-bleeding silent alimentary conditions which impair iron absorption may be the cause for IDA among premenopausal women
Ann Hematol Oncol - Volume 5 Issue 4 - 2018 ISSN : 2375-7965 | www.austinpublishing group.com Islam et al. (c) All rights are reserved
Citation: Islam MS, Dayley D and Thanigaikumar M. The Cost-effective Usefulness of Oral Iron Absorption TestProspective Evaluation in Premenopausal Women with Newly Diagnosed Iron Deficiency Anemia. Ann Hematol Oncol. 2018; 5(4): 1201 


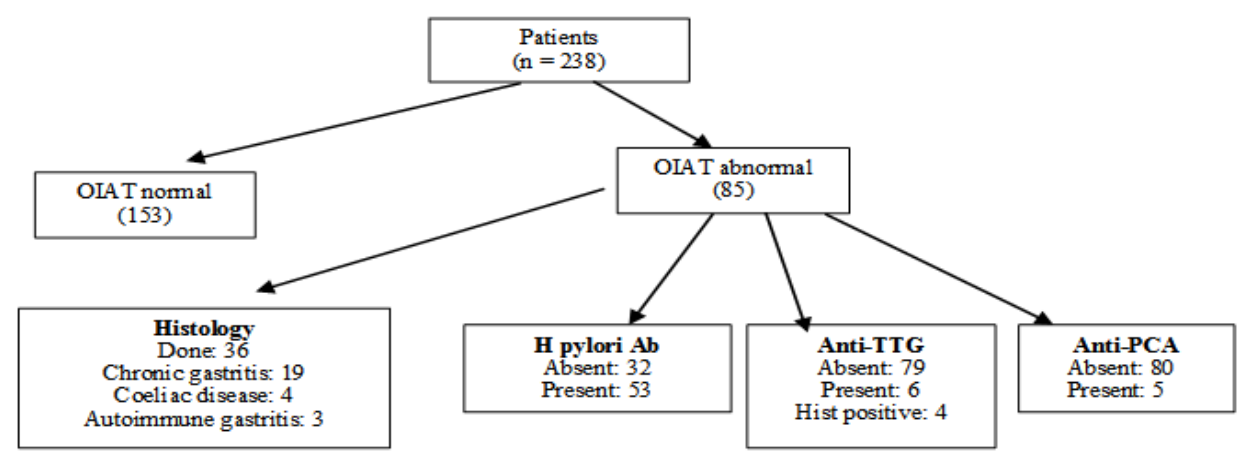

Figure 1: Patient subgroup: investigations and outcome.

OIAT: oral Iron Absorption Test; TTG, anti-TTG: Anti Tissue Transglutaminase Antibody; anti-PCA: anti-parietal cell Antibody; Hist: Histology; Ab: Antibody

[10]. The above conditions include subclinical forms of celiac disease [11-13], Helicobacter pylori ( $H$ pylori) infection [14,15] and autoimmune (atrophic) gastritis [16]. Oral iron absorption test (OIAT) may help to identify iron malabsorption in these patients and could potentially reduce the number of invasive investigations and unsuccessful trials of oral iron therapy. However, the utility of this investigation has not been verified in large prospective trials among this group of patients.

We prospectively assessed iron absorption among premenopausal women with newly diagnosed IDA referred to the hematology clinic. The primary objective of this study was to ascertain the usefulness of OIAT in predicting upper GI tract conditions associated with impaired iron absorption. The secondary objective was to compare the costs of an OIAT based approach with that of standard endoscopic evaluation.

\section{Methods}

\section{Study design and patients}

The study was conducted in two hospitals in UK between June 2013 and December 2017. Women aged 18-50 years referred to the haematology department for evaluation of IDA were prospectively recruited to the study by consecutive sampling. Patients with an identifiable cause of iron deficiency including manifest blood loss, acute GI hemorrhage and epistaxis were not enrolled in the study. Relevant data was collected from clinical notes and electronic patient database. Details on hematological, biochemical, serological and histopathological parameters were merged with the clinical data for each patient. Ethical approval for this study was obtained from the respective institutional review boards.

\section{Exclusion criteria}

Patients with one or more of the following criteria were excluded from the study: pregnancy; raised inflammatory markers (CRP $>1$ $\mathrm{mg} / \mathrm{l}$ or ESR $>20 \mathrm{~mm} / 1^{\text {st }}$ hour); specific symptoms attributable to upper GI tract disease including dysphagia, odynophagia, heartburn, dyspepsia, nausea, vomiting, anorexia and upper abdominal pain related to meals or relieved by antacids; specific symptoms of lower GI tract pathology including hematochezia, recent change in bowel habits, diarrhea, constipation and lower abdominal pain that was colicky or relieved by defaecation; positive faecal occult blood test; known coeliac disease; severe nutritional problems including anorexia nervosa; inflammatory bowel disease; parasitic infestation; known bleeding diatheses.

\section{Study definitions}

Iron deficiency anaemia was defined by the combination of $\mathrm{Hb}<12 \mathrm{~g} / \mathrm{dl}$, mean corpuscular volume $(\mathrm{MCV})<80 \mathrm{fL}$, transferrin saturation $<16 \%$ and a serum ferritin level $<15 \mathrm{ug} / 1$ [16].

Premenopausal status was ascertained by clinical history and was defined as ongoing menstruation. The menstrual cycle was considered as normal if the duration of each cycle was between 3 and 5 days, with an interval of 25-32 days between successive cycles. Menstrual flow was expressed as days per year, according to the formula: 365 days $\mathrm{x}$ days of menses/days of menstrual intervals + days of menses = days of menses/year [17]. Accordingly, 31-61 days of menses/year represented the normal range. Heavy menstrual flow was defined as $>62$ days of menses/year.

\section{Oral iron absorption test}

OIAT was performed in the outpatient setting, as outlined in previous studies $[18,19]$. After an overnight fast, venous sample for baseline $\left(\mathrm{T}_{0}\right)$ serum iron was drawn and a single ferrous sulphate tablet $200 \mathrm{mg}$ equivalent to $65 \mathrm{mg}$ elemental iron was administered orally. Serum iron levels were rechecked at 1 hour $\left(\mathrm{T}_{1 \mathrm{~h}}\right)$ and 2 hours $\left(\mathrm{T}_{2 \mathrm{~h}}\right)$ following ingestion of the tablet. Patients had no food or drink except still water between $\mathrm{T}_{0}$ and $\mathrm{T}_{2 \mathrm{~h}}$. The difference between $\mathrm{T}_{2 \mathrm{~h}}$ and $\mathrm{T}_{0}$ was taken as the estimate of iron absorption [20].

\section{Measurement of haematological parameters}

Full blood counts (FBC) were performed on EDTA anticoagulated blood using an automated COULTER' LH 750 Hematology analyser calibrated daily with standards provided by the manufacturer. Serum iron and ferritin levels were measured by Latex agglutination method on venous blood collected in lithium heparin, using AU2700/5400/ AU5800 Beckman Coulter Chemistry analyser. The normal premenopausal female reference ranges were 59-158 ug/dl for serum iron and 15-200 ug/l for serum ferritin. The analyser units were subjected to external quality control at regular monthly intervals.

\section{Subsequent investigations}

An increase in serum iron level by at least $100 \mathrm{ug} / \mathrm{dl}$ from baseline at either $\mathrm{T}_{1 \mathrm{~h}}$ or $\mathrm{T}_{2 \mathrm{~h}}$ was regarded as evidence of adequate absorption of iron from the gut [19]. In all other patients, the OIAT was considered abnormal.

If the OIAT was normal, no further tests were performed and 
Table 1: Baseline characteristics of patients and controls.

\begin{tabular}{|c|c|c|}
\hline \multicolumn{3}{|c|}{ Patients $(\mathbf{n}=\mathbf{2 3 8})$} \\
\hline Parameters* & OIAT normal $\mathbf{n = 1 5 3 ^ { + }}$ & OIAT abnormal $\mathbf{n = 8 5}$ \\
\hline Age; years & $35(18-50)$ & $34.5(18-50)$ \\
\hline Hb level; g/dl & $9.3(6.7-11.5)$ & $8.9(6.7-11.5)$ \\
\hline MCV; fl & $68(60-78)$ & $69(60-78)$ \\
\hline Serum ferritin; mg/l & $7(2-14)$ & $8(2-14)$ \\
\hline Serum iron; ug/dl & $14.5(4-25)$ & $15.5(4-25)$ \\
\hline Menstrual flow & & 53 \\
\hline Normal & 94 & 32 \\
\hline Heavy & 59 & \\
\hline
\end{tabular}

${ }^{*}$ For all parameters except menstrual flow each numerical value is the median, with range provided in parentheses

'OIAT normal' is compared with 'OIAT abnormal

${ }^{*}$ Refers to baseline serum iron, before OIAT

the patient proceeded with oral iron supplementation. Patients with abnormal OIAT had subsequent investigations which included antiTTG, serological tests to detect $H$ pylori infection and anti G-PCA levels for all those patient and an upper GI endoscopy with biopsy on patients who agreed to have this invasive investigation. These patients were treated with intravenous iron.

All endoscopic procedures were performed by gastroenterologist after obtaining informed consent. Antral, gastric body and duodenal biopsies were collected, which were evaluated by conventional histology. IgA anti-TTG levels were assessed by ELISA (Bio-Rad Laboratories, Milan, Italy), with titres $>15 \mathrm{IU} / \mathrm{ml}$ considered as positive. IgG $H$ pylori antibodies were tested by ELISA (Biohit, Helsinki, Finland), with titres $>1.1 \mathrm{IU} / \mathrm{ml}$ considered as positive. IgG anti-PCA levels were estimated using an indirect immunofluorescence semi-quantitative antibody test (IMMCO diagnostics, Buffalo, NY, USA).

\section{Results}

The study population comprised of 238 patients, all of whom underwent the OIAT. Among them, 153 had normal and 85 had impaired OIAT respectively (Figure 1). All of the 85 patients had antiTTG antibody, $H$ pylori serology and anti G-PCA levels checked on. 36 patients with an abnormal OIAT also had Upper GI endoscopy and biopsy.

\section{Prevalence of subclinical disease among patients with impaired OIAT}

Of the 85 patients with impaired OIAT, 66 had at least one abnormal test, indicative of the presence of celiac disease, $H$ pylori infection/chronic gastritis, or autoimmune gastritis. The combined prevalence of 'disease' in this subgroup of patients was $77.6 \%$. The individual prevalence for each of these GI pathologies was as follows:

\section{Celiac disease}

6 of 85 patients had tested positive for anti-TTG, of whom 4 also had histopathological features of coeliac disease (Fig 1). Among these 6 women, 2 had coexisting IgG $H$ pylori antibody and 1 had concurrent anti-PCA. The prevalence of celiac disease was $7 \%$ in the patient with abnormal OIAT.
Table 2: GI abnormalities among patients with abnormal OIAT with respect to the menstrual flow.

\begin{tabular}{|c|c|c|}
\hline \multicolumn{3}{|c|}{ Patients with impaired OIAT (n=85) } \\
\hline Abnormal tests & $\begin{array}{c}\text { Normal menstrual flow } \\
(\mathbf{n = 5 3 )}\end{array}$ & $\begin{array}{c}\text { Heavy menstrual flow } \\
(\mathbf{n = 3 2})\end{array}$ \\
\hline Coeliac disease & $\mathbf{4}(\mathbf{7 . 5 \% )}$ & $\mathbf{2} \mathbf{( 6 . 2 \% )}$ \\
\hline Anti-TTG & 4 (3 had coeliac histology) & 2 (1 had coeliac histology) \\
\hline Coeliac histology & 3 (all had anti-TTG) & 1 (1 had anti-TTG) \\
\hline H. pylori & $\mathbf{3 3}(\mathbf{6 2 . 2 \% )}$ & $\mathbf{2 0} \mathbf{( 6 2 . 5 \% )}$ \\
\hline Chronic gastritis & $12(20)$ & $7(16)$ \\
\hline Autoimmune gastritis & $\mathbf{5}(\mathbf{9 . 4 \% )}$ & $\mathbf{2}(\mathbf{6 . 2} \%)$ \\
\hline Anti-PCA & $4(1$ had atrophic gastritis) & 1 anti PCA positive \\
\hline $\begin{array}{c}\text { Atrophic gastritis } \\
\text { histology }\end{array}$ & $2(1$ had anti-PCA $)$ & 1 (1 had anti-PCA) \\
\hline
\end{tabular}

OIAT: Oral Iron Absorption Test; anti-TTG: anti tissuetransglutaminase antibody; anti-PCA: anti Parietal Cell Antibody

\section{$\boldsymbol{H}$ pylori infection and chronic gastritis}

IgG $H$ pylori antibodies were detected in the sera of 53 patients (Figure 1). As many as 19 of 36 patients who underwent endoscopy and biopsy had histological evidence of $H$ pylori related chronic gastritis. Those patients who had histological evidence of chronic gastritis, 13 of them also had IgG H. Pylori antibody. Hence the prevalence of $H$ pylori infection among the women with abnormal OIAT was $62.3 \%$.

\section{Autoimmune gastritis}

3 of 36 patients who had biopsy showed features of autoimmune gastritis on histology (Figure 1), of which 1 had anti G-PCA. In addition, 4 other patients had anti-PCA in their sera. Thus the prevalence of autoimmune gastritis among women with impaired OIAT was $8.2 \%$.

\section{Menstrual flow of patients with an identifiable GI cause for iron deficiency}

Of the 85 women with impaired OIAT, 32 (37.6\%) had heavy menstrual flow (Table 1). Comparison between the normal and heavy menstrual flow groups showed no significant difference with respect to the prevalence of coeliac disease, $H$ pylori infection or autoimmune gastritis as shown in Table 2 .

\section{Cost modelling}

The cost of OIAT per patient was estimated as the sum of 3 separate serum iron assays $\left(\mathrm{T}_{0}, \mathrm{~T}_{1 \mathrm{~h}}\right.$ and $\left.\mathrm{T}_{2 \mathrm{~h}}\right)$ and a single ferrous sulfate tablet. Each serum iron test cost $£ 2.55$ and ferrous sulfate tablets were $£ 0.11$ each, which puts a cost of $£ 7.76$ for OIAT for each patient. The costs of serological tests were $£ 14.41$ for anti-TTG, $£ 9.16$ for $H$ pylori serology and $£ 10.37$ for anti-PCA assays. Each endoscopy with gastric and small bowel histopathological examination cost $£ 101.01$. Thus to investigate a patient with all the above serological tests and histopathology would cost $£ 134.95$.

\section{Discussion}

The investigation of IDA among premenopausal women remains controversial [21]. In this group of patients, menorrhagia is often clinically implicated as the solitary cause for iron depletion. Whereas men and postmenopausal women with unexplained IDA are routinely investigated with invasive tests including GI endoscopy, most practice 
guidelines do not recommend investigations other than iron studies for menstruating women. Studies have identified a high prevalence of occult GI causes for iron deficiency among premenopausal women [17,21-23]. It has even been proposed in one study that all premenopausal women with IDA should have endoscopic evaluation irrespective of their menstrual flow [17].

The OIAT was first described about six decades ago as a clinical method to evaluate iron homeostasis [24]. It was initially employed as a technique to distinguish iron deficiency from other causes of anaemia. In spite of being an easy and inexpensive test, its utility has been questioned because of lack of sensitivity $[25,26]$. Over the past decades, there has been a revival of interest in this test as a tool to evaluate the dynamics of absorption of iron [27-30]. Due to the lack of a consensus, the management of premenopausal women referred with IDA varies considerably between centres and clinicians. The conservative approach of therapeutic iron supplementation alone carries the risk of missing or delaying the diagnosis of subclinical GI causes of iron malabsorption. The aggressive method of investigating all patients with serological tests and GI biopsy has a major implication on financial resources, laboratory workload and unnecessary use of invasive tests. We hence explored the simple and safe OIAT as a tool to identify patients with potential GI pathologies, thereby helping to obviate the need for expensive investigations in the majority of patients who do not benefit from them.

Our analysis shows a high prevalence of occult forms of celiac disease, $H$ pylori infection and autoimmune gastritis among iron deficient premenopausal women who had an abnormal OIAT. Thus it is very likely that IDA in these women was not solely attributable to menstrual flow and reduced GI absorption of iron did play a definite contributory role.

Celiac disease is a recognised cause of IDA. The pioneering study on the presence of occult celiac disease among anaemic patients identified a prevalence of 5\% [12]. A similar analysis on anemic premenopausal women found that 32 of 483 (6\%) women had a positive celiac screen by IgA anti-endomyseal antibody serology [31]. We used a combination of anti-TTG and small bowel biopsy (albeit in a small number of the study population) to identify celiac disease among patients with impaired OIAT. This strategy has been shown to have a sensitivity of $100 \%$ [32]. The causal association of $H$ pylori infection with IDA has been clearly demonstrated. Active infection with this bacterial agent is known to impair iron absorption and its eradication by appropriate antimicrobial therapy results in reversal of IDA $[14,20]$. A large retrospective study on premenopausal women with IDA identified $H$ pylori (16\%) and celiac disease (6\%) to be relatively common [33]. Although less frequent compared to the above 2 conditions, asymptomatic autoimmune gastritis has also been identified as a significant GI cause of IDA [16,34]. The mechanism of causation of iron malabsorption has been well described in each of the above three pathological states [20,35-37].

Three prior studies have performed concurrent GI and gynaecological evaluation on iron deficient premenopausal women $[22,38,39]$. All 3 studies identified a significant subclinical GI cause for IDA in the majority of women, with a prevalence as high as $95 \%$ in a prospective series [22]. Though the above studies concluded that clinicians should have a low threshold for endoscopic evaluation in the presence of digestive symptoms, faecal occult blood or weight loss, it must be noted that patients often present with no symptoms other than anaemia and menorrhagia. We propose that OIAT should be regarded as an important clinical decision tool to delineate those women with a high likelihood of having an underlying GI cause for IDA. This will help to target the appropriate population for invasive and expensive tests.

Cost benefit analysis showed significant advantages with the OIAT based management compared to a blanket endoscopy-plusserological diagnostic approach. Assuming that patients with a normal OIAT had normal iron absorption and thus a successful outcome with therapeutic iron supplementation, the savings for each such patient would be the difference between the total cost of an endoscopy-plus-serology approach (£134.95) and the cost of OIAT (£7.76). The OIAT based approach is thus 17 -fold more economical compared to the former approach in patients with normal iron absorption.

There was no difference in the prevalence of GI abnormalities between patients who had and those who did not have a heavy menstrual flow. Menorrhagia is common among women of reproductive age, with a prevalence of $30 \%$ reported in the literature [40]. When such patients present with IDA, it would be prudent to perform OIAT as menorrhagia may not be the significant underlying cause for the iron depleted state in many of these women. If the OIAT is normal, their IDA can logically be attributed to menstrual losses and they will most likely respond to oral iron replacement. However, in the presence of an impaired OIAT, they should be followed up with endoscopy and serological tests. These are the patients likely to have refractory iron deficiency due to subclinical GI pathological states associated with iron malabsorption.

\section{Authorship}

Islam MS contributed to the conception and design of the research; Islam MS and Dayley D contributed to the acquisition and analysis of the data; Islam MS and Thanigaikumar M reviewed the literature and Islam MS drafted the manuscript with help from Thanigaikumar M. All authors critically revised the manuscript, agree to be fully accountable for ensuring the integrity and accuracy of the work, and read and approved the final manuscript.

\section{Acknowledgement}

We thank to the laboratory staffs of all the two sites.

\section{References}

1. World Health Organization. Conclusions and recommendations of the WHO consultation on prevention and control of iron deficiency in infants and young children in malaria-endemic areas. Food Nutr Bull. 2007; 28: S621-S627.

2. The Global Prevalence of Anaemia in 2011. World Health Organ. Geneva.

3. Stoltzfus RJ, Mullany L, Black RE. Iron deficiency anaemia, in Comparative Quantification of Health Risks: Global and Regional Burden of Disease Attributable to Selected Major Risk Factors. 2004; vol. 1, edited by M. Ezzati et al., pp. 163-210, World Health Organ., Geneva.

4. Kassebaum NJ, Jasrasaria R, Naghavi M, Wulf SK, Johns N, Lozano R, et al. A systematic analysis of global anemia burden from 1990 to 2010. Blood. 2014; 123: 615-624.

5. Forouzanfar MH, Alexander L, Anderson HR, Bachman VF, Biryukov S, Brauer $M$, Burnett $R$, et al. Global, regional, and national comparative 
risk assessment of 79 behavioural, environmental and occupational, and metabolic risks or clusters of risks in 188 countries, 1990-2013: A systematic analysis for the Global Burden of Disease Study 2013. Lancet. 2015; 386: 2287-2323.

6. Stoltzfus RJ. Iron deficiency: Global prevalence and consequences. Food Nutr. Bull. 2003; 24: S99-S103.

7. Vannella L, Gianni D, Lahner E, Amato A, Grossi E, Delle Fave G, Annibale B. Pre-endoscopic screening for Helicobacter pylori and celiac disease in young anemic women. World J Gastroenterol. 2009; 15: 2748-2753.

8. Goddard AF, James MW, Mclntyre AS, Scott BB; British Society of Gastroenterology. Guidelines for the management of iron deficiency anaemia. Gut. 2011; 60: 1309-1316.

9. Rockey DC, Cello JP. Evaluation of the gastrointestinal tract in patients with iron-deficiency anemia. New England J Med. 1993; 329: 1691-1695.

10. Hershko C, Hoffbrand AV, Keret D, Souroujon M, Maschler I, Monselise Y, Lahad A. Role of autoimmune gastritis, Helicobacter pylori and celiac disease in refractory or unexplained iron deficiency anemia. Haematologica. 2005 90: 585-595.

11. Barton R. Iron deficiency anaemia. Patients must be screened for coeliac disease. British Medical Journal. 1997; 314: 1759-1760.

12. Corazza GR, Valentini RA, Andreani ML, D'Anchino M, Leva MT, Ginaldi L, et al. Subclinical coeliac disease is a frequent cause of iron-deficiency anaemia Scand J Gastroenterol. 1995; 30: 153-156.

13. Dickey W, Hughes D. Prevalence of celiac disease and its endoscopic markers among patients having routine upper gastrointestinal endoscopy. Am J Gastroenterol. 1999; 94: 2182-2186.

14. Annibale B, Marignani M, Monarca B, Antonelli G, Marcheggiano A, Martino G et al. Reversal of iron deficiency anemia after Helicobacter pylori eradication in patients with asymptomatic gastritis. Ann Intern Med. 1999; 131: 668-672.

15. Muhsen K, Cohen D. Helicobacter pylori infection and iron stores: a systematic review and meta-analysis. Helicobacter. 2008; 13: 323-340.

16. Marignani M, Delle Fave G, Mecarocci S, Bordi C, Angeletti S, D'Ambra G, et al. High prevalence of atrophic body gastritis in patients with unexplained microcytic and macrocytic anemia: a prospective screening study. Am J Gastroenterol. 1999; 94: 766-772.

17. Annibale B, Lahner E, Chistolini A, Gailucci C, Di Giulio E, Capurso G, et al. Endoscopic evaluation of the upper gastrointestinal tract is worthwhile in premenopausal women with iron-deficiency anaemia irrespective of menstrual flow. Scand J Gastroenterol. 2003; 38: 239-245.

18. Semrin G, Fishman DS, Bousvaros A, Zholudev A, Saunders AC, Correia $\mathrm{CE}$, et al. Impaired intestinal iron absorption in Crohn's disease correlates with disease activity and markers of inflammation. Inflamm Bowel Dis. 2006; 12: 1101-11066.

19. Alleyne M, Horne MK, Miller JL. Individualized treatment for iron-deficiency anemia in adults. Am J Medi. 2008; 121: 943-948.

20. Ciacci C, Sabbatini F, Cavallaro R, Castiglione F, Di Bella S, lovino P, et al. Helicobacter pylori impairs iron absorption in infected individuals. Dig Liver Dis. 2004 ; 36 : 455-460.

21. Vannella L, Aloe Spiriti MA, Cozza G, Tardella L, Monarca B, Cuteri A Benefit of concomitant gastrointestinal and gynaecological evaluation in premenopausal women with iron deficiency anaemia. Aliment Pharmacol Therap. 2008; 28: 422-430.
22. Kepczyk T, Cremins JE, Long BD, Bachinski MB, Smith LR, McNally PR. A prospective, multidisciplinary evaluation of premenopausal women with irondeficiency anemia. Am J Gastroenterol. 1999; 94: 109-115.

23. Bini EJ, Micale PL, Weinshel EH. Evaluation of the gastrointestinal tract in premenopausal women with iron deficiency anemia. Am J Med. 1998; 105: 281-286.

24. Josephs HW. Absorption of iron as a problem in human physiology; a critica review. Blood. 1958; 13: 1-54.

25. Crosby WH, O'Neil-Cutting MA. A small-dose iron tolerance test as an indicator of mild iron deficiency. J AMA. 1984; 251: 1986-1987.

26. Verloop MC, Meeuwissen JE, Blokhuis EW. Comparison of the iron absorption test with the determination of the iron-binding capacity of serum in the diagnosis of iron deficiency. Br J Haematol. 1958; 4: 70-81.

27. Joosten E, Vander Elst B, Billen J. Small-dose oral iron absorption test in anaemic and non-anaemic elderly hospitalized patients. Eur J Haematol. 1997; 58: 99-103.

28. Bastani B, Islam S, Boroujerdi N. Iron absorption after single pharmacological oral iron loading test in patients on chronic peritoneal dialysis and in healthy volunteers. Perit Dial Int. 2000; 20: 662-666.

29. Hoppe M, Hulthen L, Hallberg L. The validation of using serum iron increase to measure iron absorption in human subjects. Br J Nutr. 2004; 92: 485-488.

30. Jensen NM, Brandsborg M, Boesen AM, Yde H, Dahlerup JF. Low-dose oral iron absorption test: establishment of a reference interval. Scand J Clin Lab Invest. 1998; 58: 511-519.

31. Unsworth DJ, Lock RJ, Harvey RF. Improving the diagnosis of coeliac disease in anaemic women. Br J Haematol. 2000; 111: 898-901.

32. Hopper AD, Cross SS, Hurlstone DP, McAlindon ME, Lobo AJ, Hadjivassiliou $\mathrm{M}$, et al. Pre-endoscopy serological testing for coeliac disease: evaluation of a clinical decision tool. BMJ. 2007; 334: 729 .

33. Carter D, Maor Y, Bar-Meir S, Avidan B. Prevalence and predictive signs for gastrointestinal lesions in premenopausal women with iron deficiency anemia. Dig Dis Sci. 2008; 53: 3138-3144.

34. Hershko C, Ronson A, Souroujon M, Maschler I, Heyd J, Patz J. Variable hematologic presentation of autoimmune gastritis: age-related progression from iron deficiency to cobalamin depletion. Blood. 2006; 107: 1673-1679.

35. Rad R, Schmid RM, Prinz C. Helicobacter pylori, iron deficiency, and gastric autoimmunity. Blood. 2006; 107: 4969-4970

36. Hershko C, Skikne B. Pathogenesis and management of iron deficiency anemia: emerging role of celiac disease, helicobacter pylori, and autoimmune gastritis. Semin Hematol. 2009; 46: 339-350.

37. Annibale B, Capurso G, Delle Fave G. The stomach and iron deficiency anaemia: a forgotten link. Dig Liver Disease. 2003; 35: 288-295.

38. Vannella L, Aloe Spiriti MA, Cozza G, Tardella L, Monarca B, Cuteri A, et al. Benefit of concomitant gastrointestinal and gynaecological evaluation in premenopausal women with iron deficiency anaemia. Aliment Pharmacol Ther. 2008; 28: 422-430.

39. Green BT, Rockey DC. Gastrointestinal endoscopic evaluation of premenopausal women with iron deficiency anaemia. J Clin Gastroenterol. 2004; 38: 104-109

40. El-Hemaidi I, Gharaibeh A, Shehata H. Menorrhagia and bleeding disorders. Curr Opinion in Obstetrics and Gynecology. 2007; 19: 513-520.
Ann Hematol Oncol - Volume 5 Issue 4 - 2018 ISSN : 2375-7965 | www.austinpublishing group.com Islam et al. (C) All rights are reserved
Citation: Islam MS, Dayley D and Thanigaikumar M. The Cost-effective Usefulness of Oral Iron Absorption TestProspective Evaluation in Premenopausal Women with Newly Diagnosed Iron Deficiency Anemia. Ann Hematol Oncol. 2018; 5(4): 1201 